\title{
Edge-Weighted Contact Representations of Planar Graphs
}

\author{
Martin Nöllenburg, Roman Prutkin, and Ignaz Rutter \\ Institut für Theoretische Informatik, Karlsruhe Institute of Technology, Germany
}

\begin{abstract}
We study contact representations of edge-weighted planar graphs, where vertices are rectangles or rectilinear polygons and edges are polygon contacts whose lengths represent the edge weights. We show that for any given edge-weighted planar graph whose outer face is a quadrangle, that is internally triangulated and that has no separating triangles we can construct in linear time an edge-proportional rectangular dual if one exists and report failure otherwise. For a given combinatorial structure of the contact representation and edge weights interpreted as lower bounds on the contact lengths, a corresponding contact representation that minimizes the size of the enclosing rectangle can be found in linear time. If the combinatorial structure is not fixed, we prove NP-hardness of deciding whether a contact representation with bounded contact lengths exists.

Finally, we give a complete characterization of the rectilinear polygon complexity required for representing biconnected internally triangulated graphs: For outerplanar graphs complexity 8 is sufficient and necessary, and for graphs with two adjacent or multiple non-adjacent internal vertices the complexity is unbounded.
\end{abstract}

\section{Introduction}

Representing graphs by intersections or contacts of geometric objects has a long history in graph theory and graph drawing, which is covered in monographs and surveys [12,21]. For example, Koebe's circle packing theorem from 1936 establishes that every planar graph has a contact representation by touching disks (and vice versa) [15]; more recently it was shown that every planar graph is the intersection graph of line segments [8].

In this paper we are interested in a special class of contact representations for plane graphs, namely hole-free side-contact representations using rectangles and rectilinear polygons. In a rectilinear representation of a plane graph $G=(V, E)$ every vertex $v \in V$ is represented as a simple rectilinear polygon $P(v)$ and there is an edge $e=u v \in E$ if and only if $P(u)$ and $P(v)$ have a non-trivial common boundary or contact path $s(e)$ (i.e., length $|s(e)|>0)$. It is further required that the union $\bigcup_{v \in V} P(v)$ forms a simple rectilinear polygon itself, i.e., there are no holes in the representation. A standard assumption, which we will make throughout this paper, is that $G$ is an internally triangulated plane graph. This excludes the degenerated case of four polygons that meet in a single point. A rectangular dual [16] of a graph $G$ is a dissection of a rectangle into rectangles, which represents $G$ as a contact graph; rectangular duals are thus an interesting special case of rectilinear representations, where all polygons and their union are rectangles. Rectangular duals and rectilinear representations with low-complexity polygons have practical applications, e.g., in VLSI design, cartography, or floor planning and surveillance in 
buildings [22]. In these applications, the area of vertex polygons and/or the boundary length of adjacent polygons often play an important role, which immediately raises the question of representing weighted graphs whose weights control these parameters.

Previously, rectilinear representations and rectangular duals have been studied only for unweighted graphs [16, 19] and vertex-weighted graphs [2,5,10], where the polygon areas must be proportional to the vertex weights. This paper covers the remaining open aspect of representing edge-weighted graphs as touching rectilinear polygons. A natural way of encoding edge weights in a rectilinear representation is to require that the contact lengths of all adjacent vertex polygons are proportional to the given edge weights. So we define an edge-proportional rectilinear representation (EPRR) of an edge-weighted graph $\left(G, \omega: E \rightarrow \mathbb{R}^{+}\right)$as a rectilinear representation in which additionally the contact length $|s(e)|=\omega(e)$ for every edge $e \in E$.

Related work. It is known that unweighted graphs always have a rectilinear representation using rectangles, L-shaped and T-shaped polygons, i.e., at most 8-gons, and that there are some graphs for which complexity 8 is necessary [19,24]. The class of unweighted graphs that have a rectangular dual is characterized as all plane triangulations without separating triangles [16, 18]. Orientation-constrained rectangular duals have also been considered [10].

For vertex-weighted graphs the goal is to find area-proportional rectilinear representations, in which the area of a polygon $P(v)$ is proportional to the weight of vertex $v$. In a series of papers the polygon complexity that is sufficient to realize any weighted graph was decreased from 40 corners [4] over 34 corners [13], 12 corners [6], 10 corners [1] down to 8 corners [2], which is best possible due to the earlier lower bound of 8 [24]. Weighted rectangular duals have also been studied before, e.g., van Kreveld and Speckmann [17] presented several algorithms to compute rectangular duals with low area error. Eppstein et al. [10] gave a necessary and sufficient condition for rectangular duals to be area-universal, i.e., rectangular duals that can realize arbitrary vertex weights without changing their combinatorial structure. They also showed that for a given combinatorial structure of the dual and given vertex weights it can be efficiently tested whether these weights can be represented as the perimeters of the vertex rectangles rather than their areas. Biedl and Genc [5] showed that testing whether a rectangular representation with prescribed areas exists is NP-hard if the complexity of the outer face is unbounded.

Drawing planar graphs with edge weights as standard node-link diagram, where edge lengths are proportional to the edge weights is an NP-hard problem [9] but can be decided in linear time for planar 3-connected triangulations [7].

Contribution. In Section 2 we consider rectangular duals. We present a linear-time algorithm that decides whether a given graph $G$ has an edge-proportional rectangular dual (EPRD) and constructs it in the positive case (Section 2.1). Moreover, if the combinatorial structure of the dual is specified, we use existing tools to find a rectangular dual where $|s(e)| \geq \omega(e)$ for all $e \in E$ and the size of the outer rectangle is minimum (Section 2.2). On the other hand, without a fixed combinatorial structure, we prove NP-hardness of the problem to find a representation where the lengths of the contact segments are lower and upper bounded (Section 2.3). 
In Section 3, we consider EPRRs and show that for representing outerplanar graphs polygon complexity 8 is sometimes necessary and always sufficient. The class of outerpillars (outerplanar graphs whose weak dual is a caterpillar, i.e., a tree for which a path remains after removing all leaves) always has EPRRs of complexity 6, but already outerlobsters (outerplanar graphs whose weak dual is a lobster, i.e., a tree for which a caterpillar remains after removing all leaves) require complexity 8 . If, on the other hand, the graph has two adjacent or multiple non-adjacent internal vertices, polygons of unbounded complexity are sometimes necessary. This completely characterizes the complexity of EPRRs for internally triangulated graphs.

\section{Rectangular Duals with Contact Length Specifications}

\subsection{Rectangular Duals with Fixed Contact Lengths}

He [11] proved that a a planar graph $G$ has a rectangular dual with four rectangles on the boundary if and only if (1) every interior face of $G$ is a triangle and the outer face is a quadrangle, and (2) $G$ has no separating triangles. We call a graph satisfying these conditions properly triangular planar $(P T P)$. Moreover, we denote the four vertices on the boundary of the outer face by $v_{N}, v_{W}, v_{S}$ and $v_{E}$ in counterclockwise order.

A rectangular dual $R$ of a PTP graph $G=(V, E)$ defines an orientation and a partition of the internal edges of $G$ into two sets $T_{1}$ and $T_{2}$. The set $T_{1}$ contains the edges $e$ for which $s(e)$ is horizonal, the remaining edges are in $T_{2}$. The orientation is obtained by orienting $u v \in T_{1}$ from $u$ to $v$ if $R(u)$ is below $R(v)$, similarly $u v \in T_{2}$ is oriented from $u$ to $v$ if $R(u)$ is to the left of $R(v)$. For a vertex $v$ and one of the partitions $T_{i}, i=1,2$, we denote by $T_{i}^{\leftarrow}(v)$ and $T_{i} \rightarrow(v)$ the incoming and outgoing edges of $v$ that are contained in $T_{i}$, respectively. The orientation and partition then satisfies the following properties.

1. For each vertex $v$, a counterclockwise enumeration of its incident edges starting with the rightmost edge in $T_{1}^{\rightarrow}(v)$ encounters first the edges in $T_{1}^{\rightarrow}(v)$, then in $T_{2}^{\leftarrow}(v)$, then in $T_{1}^{\leftarrow}(v)$ and finally in $T_{2}^{\rightarrow}(v)$, and

2. all interior edges incident to $v_{N}, v_{W}, v_{S}$ and $v_{E}$ are in $T_{1}^{\leftarrow}\left(v_{N}\right), T_{2}^{\rightarrow}\left(v_{W}\right), T_{1}^{\rightarrow}\left(v_{S}\right)$ and $T_{2}^{\leftarrow}\left(v_{E}\right)$, respectively.

We call any partition and orientation of the edges satisfying these properties a regular edge labeling (REL). In his work, He [11] showed that every PTP graph admits a REL, and that a corresponding rectangular dual can be constructed from a REL in linear time.

It is not hard to see that a REL derived from an EPRD has additional properties, following from the fact that for each rectangle the total length of the contacts on the left and right side as well as on the top and bottom side are the same, respectively.

$$
\sum_{e \in T_{1}^{\leftarrow}(v)} \omega(e)=\sum_{e \in T_{1}^{\rightarrow}(v)} \omega(e), \sum_{e \in T_{2}^{\leftarrow}(v)} \omega(e)=\sum_{e \in T_{2}^{\rightarrow}(v)} \omega(e) .
$$

We call any REL satisfying this condition an edge proportional REL (EPREL). In the following we show that a weighted PTP graph $G=(V, E)$ has a unique EPREL, if one exists. Moreover, we show how to test the existence of such an EPREL in linear time and how to construct a corresponding EPRD. 


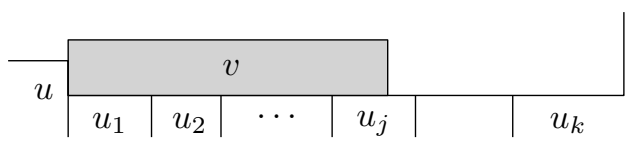

Fig. 1. Prior to the insertion of the next inner rectangle there is always a U-shape for which there exists a vertex whose corresponding rectangle needs to be inserted at the lower left corner of the U-shape in a unique way.

Lemma 1. For an inner vertex $v$, any one of the sets $T_{1}^{\leftarrow}(v), T_{1}^{\rightarrow}(v), T_{2}^{\leftarrow}(v)$ or $T_{2} \rightarrow(v)$ of an EPREL completely fixes the orientation and the partition of the edges incident to $v$. A corresponding orientation and partition can be found in $O(\operatorname{deg}(v))$ time if it exists.

Proof. Assume $T_{1}^{\leftarrow}(v)$ is known, the other cases are symmetric. Let $\omega_{1}=\sum_{e \in T_{1}^{\leftarrow}(v)} \omega(e)$ and let further $\omega_{2}=\left(\sum_{u v \in E} \omega(u v)-2 \omega_{1}\right) / 2$. It follows from condition (1) that necessarily $\sum_{e \in T_{2} \leftarrow(v)}=\sum_{e \in T_{2} \rightarrow(v)}=\omega_{2}$. Due to the requirement of the REL for the ordering of the edges around $v$, there is at most one way to orient and partition the edges incident to $v$ such that condition (1) holds. It can be found in $O(\operatorname{deg}(v))$ time by a simple counterclockwise traversal of the edges incident to $v$, starting from the last edge in the known set $T_{1}^{\leftarrow}(v)$.

Observe that if the partition and orientation of the edges incident to a vertex $v$ is determined, the shape of the rectangle representing $v$ is completely fixed. Moreover, the conditions on the edges incident to $v_{N}, v_{W}, v_{S}$ and $v_{E}$ completely specify a rectangle $R_{I}$ into which the remaining rectangles have to be inserted. We construct an ordering of the internal vertices $v_{1}, \ldots, v_{n-4}$ such that we can iteratively apply Lemma 1 to determine uniquely the shape of their rectangle as well as the position where they have to be inserted in $R_{I}$. Since we are completely guided by necessary conditions, this either results in a correct EPRD, or the procedure fails at some point, in which case an EPRD does not exist.

We maintain the following invariants in each step $i$.

1. The position and dimension of $R\left(v_{1}\right), \ldots, R\left(v_{i}\right)$ are uniquely determined.

2. All contacts between already inserted rectangles or the boundary polygon $R_{I}$ have correct lengths.

3. The upper boundary of the polygon $\bigcup_{j=1}^{i} R\left(v_{j}\right) \cup R\left(v_{S}\right) \cup R\left(v_{W}\right) \cup R\left(v_{E}\right)$ is an $x$ monotone chain.

Note that initially $i=0$ and all properties hold. By the third property there exists a $U$-shape on the upper boundary whose bottom side is horizontal, i.e., there are two vertical segments adjacent to and above the bottom side. Let $u$ be the lowest rectangle bounding this U-shape from the left and let $u_{1}, \ldots, u_{k}$ denote the rectangles bounding the U-shape from below; see Fig. 11 The corner at $R(u)$ and $R\left(u_{1}\right)$ implies that if $G$ admits an EPRD, then there exists a unique vertex $v$ that is not yet inserted, and that is incident to both $u$ and $u_{1}$. We choose this vertex as the next vertex $v_{i}$. Its adjacencies to the vertices $u_{1}, \ldots, u_{j}$ for some $j \leq k$ completely determine its contacts from below, and hence $T_{1}^{\leftarrow}(v)$. By Lemma 1 its shape is completely determined. Moreover, the position is fixed as well due to the corner between $R(u)$ and $R\left(u_{1}\right)$. This implies Invariant 1. 
Invariant 2 is either satisfied or an EPRD does not exist since we only followed necessary conditions. Finally, Invariant 3 holds due to the choice of the U-shape. The whole algorithm can be implemented to run in linear time.

Theorem 1. For an edge-weighted PTP graph $G$ there exists at most one EPRD. It can be computed in linear time if it exists.

\subsection{Rectangular Duals with Minimum Contact Lengths}

Next we consider a slightly relaxed version of the problem, where we assume that the input consists of a REL, which combinatorially describes the rectangular dual, and a weight function specifying minimum contact lengths for all edges. The task is then to find a rectangular dual according to the given REL that minimizes the total size of the layout. Note that in this setting any instance is feasible since any given rectangular dual can be scaled to become a feasible solution.

Using the method of He [11] we can construct in linear time a rectangular dual $R$ of the PTP graph $G$ that realizes the given REL, but does not yet satisfy the edge-length constraints. We can either modify He's algorithm to directly compute a suitable RD in linear time, or take a slightly different perspective on the problem. The rectangular dual $R$ of $G$ can also be seen as an orthogonal representation with rectangular faces of the dual graph $G^{\star}$ of $G$, where every degree-3 vertex corresponds to a face of $G$ and every orthogonally drawn edge corresponds to two adjacent faces. This allows us to use a modified version of a linear-time compaction algorithm for orthogonal drawings [3, Chapter 5.4] that respects the minimum contact length $\omega(e)$ for each $e \in E$ as the minimum length of the corresponding dual edge $e^{\star}$. The main idea of the approach is to define two independent planar edge-weighted st-graphs $N_{\text {hor }}$ and $N_{\text {ver }}$, the first one using the edges in $T_{1}$, the other one the edges in $T_{2}$. Tamassia [23] described an algorithm to compute two weighted topological numberings on $N_{\text {hor }}$ and $N_{\text {ver }}$ from which the coordinates of all vertices of $R$ (or $G^{\star}$ ) can be obtained. These numberings immediately minimize the total height, total width and area of $R$ subject to the length constraints.

Theorem 2. For a weighted PTP graph $(G, \omega)$ with a given REL, a corresponding rectangular dual with minimum width, height, and area of the inner rectangles can be computed in linear time such that each edge e is represented by a contact of length at least $\omega(e)$.

In particular, if the given REL is an EPREL, the algorithm computes an EPRD.

Corollary 1. A weighted PTP graph admits an EPREL if and only if it admits an EPRD.

\subsection{Rectangular Duals with Minimum and Maximum Contact Lengths}

Unlike in the case of precisely specified or lower-bounded contact lengths covered in the previous sections, the problem becomes NP-hard if we are given lower and upper bounds for the contact lengths and need to decide the existence of a rectangular dual respecting these bounds. 


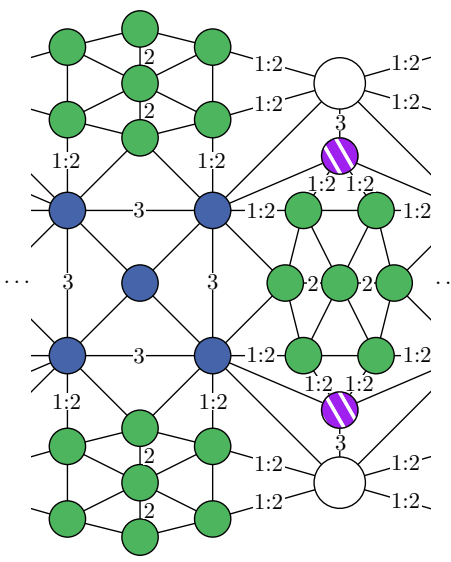

(a)

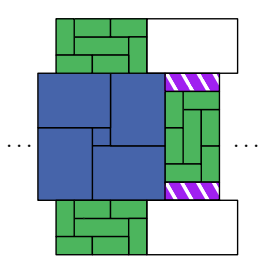

(b)

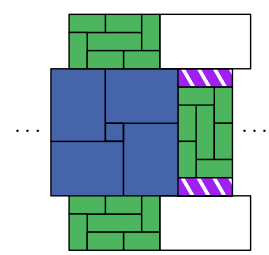

(c)

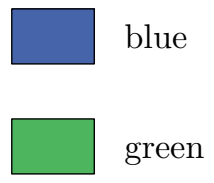

purple

Fig. 2. Basic building block consisting of a 5-vertex graph (blue), three 7-vertex graphs (green), and four additional vertices (white and purple). The edge weights in (a) are $\alpha(e)=\beta(e)=d$ for an edge $e$ labeled with a single value $d$ (with $d=1$ for unlabeled edges) and $\alpha(e)=1, \beta(e)=2$ for the label $1: 2$. Figures (b) and (c) show the only two valid rectangle realizations.

Theorem 3. Given a PTP graph $G=(V, E)$ with two edge-weight functions $\alpha, \beta: E \rightarrow$ $\mathbb{R}^{+}$with $\alpha(e) \leq \beta(e)$ for all $e \in E$, it is NP-hard to decide if $G$ has a rectangular dual $\mathcal{R}=\{R(v) \mid v \in V\}$ so that for every edge $u v \in E$ the contact segment $s(e)$ has length $\alpha(e) \leq|s(e)| \leq \beta(e)$.

Proof. The proof is a gadget proof reducing from the NP-complete problem PLANAR 3SAT [20]. PlanAR 3SAT is the satisfiability problem for Boolean formulae $\phi$ in conjunctive normal form with at most three variables per clause, which are planar in the way that the induced bipartite variable-clause graph $H_{\phi}$ consisting of a vertex for every variable, a vertex for every clause, and an edge for every occurrence of a variable in a clause is planar. Such a graph $H_{\phi}$ can be drawn on a grid of polynomial size with all variable vertices placed on a horizontal line and the clause vertices connected in a comb-shaped manner from above or below that line [14]. In our reduction, we create an edge-weighted PTP graph $G_{\phi}$ for a planar 3Sat formula $\phi$, which has a rectangular dual mimicking the above mentioned drawing of $H_{\phi}$ if and only if $\phi$ is satisfiable.

The basic building block for the variable gadgets and their links to the clause gadgets is a 5-vertex graph flanked by three auxiliary isomorphic 7-vertex graphs, see Figure 2 . The important property of this subgraph is that it has only two valid realizations as a rectangle contact graph, one of which encodes the value true, the other one the value false, and both of which have the same outer shape. Any other attempt to realize this subgraph violates either the edge length constraints or requires non-rectangular vertex regions. Here we omit the detailed arguments due to space restrictions.

Several copies of the building block can be attached to each other both vertically and horizontally so that the green 7-vertex subgraphs link two adjacent blue 5-vertex subgraphs. This synchronizes the states of all blocks: either all linked blocks are in the true 


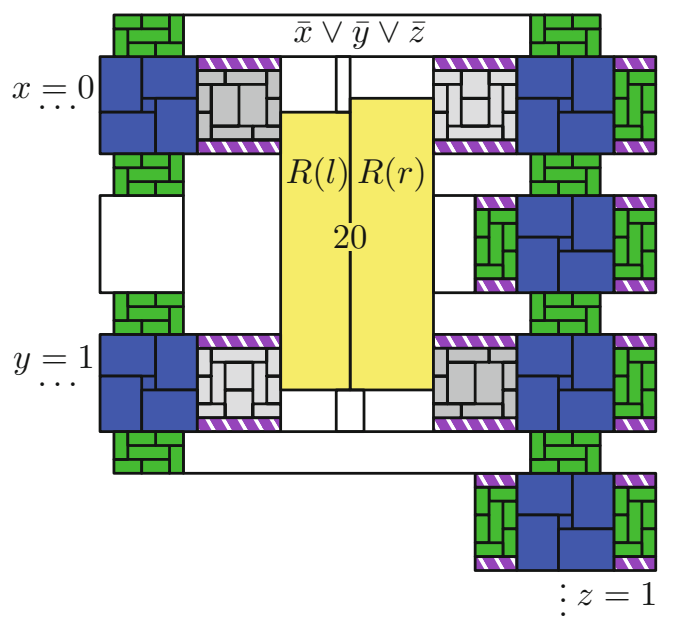

Fig. 3. Clause gadget for the clause $\bar{x} \vee \bar{y} \vee \bar{z}$ in the state $x=0, y=1, z=1$. The contact length $|s(l r)|$ of the two yellow rectangles $R(l)$ and $R(r)$ is 20 .

state or all are in the false state. This allows us to create horizontal variable gadgets with vertical branches leading towards the clause gadgets. Two different variable gadgets are separated by three buffer vertices (or rectangles) that do not link the gadget states.

It remains to describe the clause gadget, whose rectangular layout is shown in Figure 3. It takes three inputs, two from the left side and one from below or above depending on whether the clause gadget is placed above or below the variable row. Note that the input from below or above is duplicated. Each input port consists either of an inverter gadget (light gray in Figure 3), which inverts the state of the incoming truth value or a replicator gadget (dark gray in Figure 3), which simply copies the incoming truth value. The type of the port gadget depends not only on whether the literal in the clause is positive or negative, but also on the position of the port in the clause gadget: The top left and the bottom right ports use an inverter for a positive literal and a replicator for a negative one; the bottom left and top right ports use a replicator for a positive literal and an inverter for a negative one. This configuration has the following effect on the two core rectangles $R(l)$ and $R(r)$ of the clause gadget, whose contact length is bounded by $\alpha(l r)=19$ and $\beta(l r)=20$. Every false literal stretches its adjacent rectangle $R(l)$ or $R(r)$ by a length of 1 (in fact by a length of 2 for $R(r)$ since the last literal is duplicated). If all literals are true then both $R(l)$ and $R(r)$ have height 19 and also $|s(l r)|=19$. By inspecting all cases one can see that as long as one literal is true we have $19 \leq|s(\operatorname{lr})| \leq 20$, but as soon as all three literals are false the contact length becomes $|s(l r)|=21$ violating the specified upper bound. This is exactly the behavior needed for the reduction.

In order to create an actual PTP graph, the remaining gaps between the described gadgets must be filled by dummy rectangles, i.e., dummy vertices in $G_{\phi}$. Since $H_{\phi}$ can be drawn on a grid of polynomial size it is clear that $G_{\phi}$ also has polynomial size. 
A variant of the problem with lower bounded contact lengths and upper bounded rectangle areas turns out to be NP-hard as well as the next theorem shows. The proof is very similar to the proof of Theorem 3 and omitted due to space constraints.

Theorem 4. Given a PTP graph $G=(V, E)$ with an edge-weight function $\omega: E \rightarrow \mathbb{R}^{+}$ and a vertex-weight function $\gamma: V \rightarrow \mathbb{R}^{+}$, it is $N P$-hard to decide if $G$ has a rectangular dual $R=\{R(v) \mid v \in V\}$ so that

(i) for every edge $e \in E$ the contact segment $s(e)$ has length $|s(e)| \geq \omega(e)$ and

(ii) for every vertex $v \in V$ the rectangle $R(v)$ has area $|R(v)| \leq \gamma(v)$.

\section{Length-Universal Rectilinear Layouts}

In this section we consider the number of bends required for constructing edgeproportional rectilinear representations (or EPRRs for short) of internally triangulated planar graphs $G=(V, E, \omega)$. In our proofs we assume that the graphs are biconnected because every internally triangulated graph can be made biconnected by adding vertices in the outer face. Since our representations preserve the outerplanar embedding, the removal of the corresponding polygons does not create holes.

The complexity of a rectilinear polygon $p$ is its number of bends, and is denoted by $k(p)$. The complexity of an EPRR $P$ is $k(P):=\max _{u \in V} k(P(u))$. The complexity of a graph $G$ with weight function $\omega$ is $k(G, \omega)=\min _{P \in \mathcal{P}} k(P)$, where $\mathcal{P}$ denotes all EPRRs of $(G, \omega)$. For a graph class $\mathcal{G}$, the complexity is the maximum complexity for any graph from $\mathcal{G}$ with any weight function $\omega$, i.e., $k(\mathcal{G})=\max _{G \in \mathcal{G}} \max _{\omega: E \rightarrow \mathbb{R}^{+}} k(G, \omega)$. We are interested in determining $k(\mathcal{G})$ for different classes of graphs.

Theorem 5. For any biconnected internally triangulated graph $G$ with two adjacent internal vertices and any positive integer $k_{0}$, there exists a weight function $\omega$ such that $k(G, \omega) \geq k_{0}$.

Let $\mathcal{I}$ denote the class of biconnected internally triangulated graphs containing no adjacent internal vertices; we have $k(\mathcal{I}) \geq k_{0}$ for any positive integer $k_{0}$.

Proof. For the first part of the theorem let $G$ be a biconnected internally triangulated graph with two adjacent internal vertices $u$ and $v$. We define $\omega$ such that $\omega(e)=1$ for all $e \neq u v$ and $\omega(u v)=k_{0} \cdot(\operatorname{deg}(u)+\operatorname{deg}(v)-2)=: M$. Now, in any contact representation the polygon $P(u) \cup P(v)$ has a boundary of length $\operatorname{deg}(u)+\operatorname{deg}(v)-2$. On the other hand, this polygon necessarily contains the contact path of length $M$ corresponding to the edge $u v$. This path has at least $M /(\operatorname{deg}(u)+\operatorname{deg}(v)-2)=k_{0}$ bends as it would cross the boundary of $P(u) \cup P(v)$ otherwise.

For the second part, consider the graph $K_{4}$ with internal vertex $x$, outer vertices $a, b, c$ and all edge weights set to 1 except for $\omega(a x)=2$. Since $\omega(a x) \geq \omega(b x)+\omega(c x)$ the path $s(a x)$ must have a bend. Now consider a fan-graph on $k_{0}+2$ vertices with center vertex $a$, and insert into each fan triangle $T$ a new internal vertex $x_{T}$ connected with edges of weight 2 to $a$ and weight 1 to the other two vertices of $T$. By the above observation the polygon $P(a)$ needs one bend per path $s\left(a x_{T}\right)$ for all $k_{0}$ fan triangles $T$.

This shows that to achieve positive results, we may allow only few isolated interior vertices. Thus we consider outerplanar graphs and graphs with one internal vertex. 


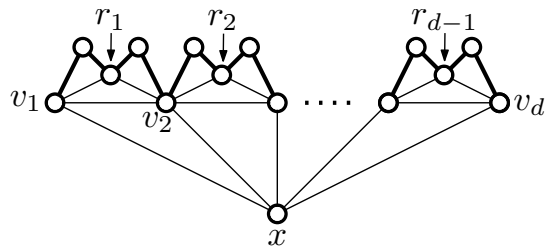

(a)

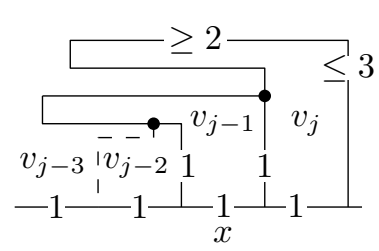

(b)

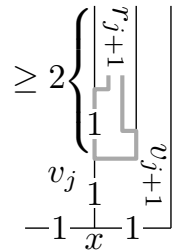

(c)

Fig. 4. A graph that does not admit an EPRR with 6-gons for $d=55$; thin edges have weight 1 and thick edges have weight 4

Proposition 1. For the class $\mathcal{O}$ of biconnected internally triangulated outerplanar graphs $k(\mathcal{O}) \geq 8$.

Proof. Consider the family of graphs depicted in Figure 4a We show that if all thin edges have weight 1 and the thick edges have weight 4 , then for $d=55$, the corresponding weighted graph $(G, \omega)$ does not admit a representation with complexity less than 8 . Assume for contradiction that $P$ is a representation with complexity at most 6 . (Note that the complexity of a rectilinear polygon is always even.)

Claim. There exists a chain $Q=\left\{v_{i}, \ldots, v_{i+8}\right\}$ such that the contacts between $P(x)$ and $P(v)$ with $v \in Q$ all lie on a common line.

This follows easily from the fact that there are 55 vertices on the path $v_{1}, \ldots, v_{d}$, and to avoid all such chains $Q, P(x)$ would need to bend at least once every 9th contact. But then we get at least $\lceil 55 / 9\rceil=7$ bends on $P(x)$.

Claim. Let $i$ and $Q$ be chosen as in the previous claim, and without loss of generality assume that their contacts lie on a common horizontal line. Then for $j=i+3, \ldots, i+5$, we have that $P\left(v_{j}\right)$ has height more than 3 .

Assume for a contradiction that the height of $P\left(v_{j}\right)$ is at most 3. Then, as $P\left(v_{j}\right)$ has perimeter at least $2 \cdot 4+5=13$, it must be realized as an L-shape with an overhang of width at least 2 , say to the left. It follows that $P\left(v_{j-1}\right)$ has height at most 1 ; see Fig. $4 \mathrm{~b}$ But then $P\left(v_{j-2}\right)$ has perimeter at most 4 as it is enclosed in a $1 \times 1$-box, a contradiction. The case that the overhang is to the right is symmetric. This proves the second claim.

Now consider $P\left(v_{i+4}\right)$. Either its left or right side does not have a bend, and hence is a vertical segment of length at least 3 . Without loss of generality assume that it is the right side. We then consider $v_{i+4}$ and $v_{i+5}$, and their common neighbor $r_{i+4}$. The situation is depicted in Fig. 4c. The path $s\left(r_{i+4} v_{i+5}\right)$ has length 1, and thus bends at the reflex point of $P\left(v_{i+5}\right)$. Since both $P\left(v_{i+4}\right)$ and $P\left(v_{i+5}\right)$ have height at least 3 and $P\left(r_{i+4}\right)$ has perimeter at least $10, P\left(r_{i+4}\right)$ needs two bends in order to achieve the correct contact lengths with both of them; a contradiction to the assumption that $P$ has complexity 6 .

On the other hand, we describe an algorithm that produces for any outerplanar graph $G$ with weight function $\omega$ a representation with complexity 8 .

Proposition 2. For the class $\mathcal{O}$ of internally triangulated outerplanar graphs $k(\mathcal{O}) \leq 8$. 


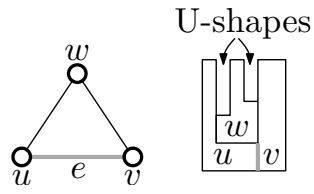

(a)

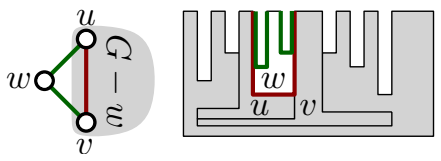

(b)

Fig. 5. Construction of a rectilinear representation by 8-gons for an outerplanar graph. (a) A triangle with reference edge $e$, forming the base case. (b) Inserting $P(w)$ into the U-shape of $u v$ (red), creating new U-shapes for $u w$ and $v w$ (green).

Proof. We show that for any biconnected outerplanar graph $G=(V, E)$ with weight function $\omega$ and a reference edge $e \in E$ on the outer face, there exists an EPRR $P$ such that for each edge $u v$ on the outer face with $u v \neq e$, there exists a U-shape whose left and right boundary are formed by the polygons $P(u)$ and $P(v)$, whose open side points to the top, and whose width is at most $\varepsilon / 2$, where $\varepsilon$ is the smallest weight of all edges.

For a triangle $u v w$ with reference edge $u v$ this is obviously possible; see Fig. 5a. We construct the drawing inductively. Let $G$ be an arbitrary graph with reference edge $e$. Since $G$ is outerplanar and has more than three vertices, it has a degree-2 vertex $w$ that is not adjacent to $e$. By induction $G-w$ has a desired representation $P$ with respect to the reference edge $e$. Let $u$ and $v$ denote the two neighbors of $w$, which are connected by an edge on the outer face of $G-w$. Note that the presence of edge $u v$ implies that $G-w$ remains biconnected. By the properties of $P$, there is a $\mathrm{U}$-shape for the edge $u v$. We then insert a new polygon $P(w)$ into this U-shape as illustrated in Fig. 5b Obviously, this preserves all invariants.

Propositions 1 and 2 imply that for the class $\mathcal{O}$ of outerplanar graphs, we have $k(\mathcal{O})=8$. We remark that our technique for representing outerplanar graphs with 8-gons extends to graphs with a single internal vertex, by wrapping the drawing around this central vertex while creating the U-shapes for the outer edges. Moreover, in the outerplanar case, it is simultaneously possible to achieve given areas for all vertices by suitably stretching the polygons to satisfy the area demands. Next, we consider special cases and show that outerpaths (outerplanar graphs whose weak dual is a path) require six bends, and that six bends suffice for outerpillars. The proof of the next proposition is omitted.

Proposition 3. For the class $\mathcal{P}$ of internally triangulated outerpaths $k(\mathcal{P}) \geq 6$.

Proposition 4. For the class $\mathcal{P}^{\prime}$ of internally triangulated outerpillars $k\left(\mathcal{P}^{\prime}\right) \leq 6$.

Proof. Let $G^{\prime}$ be an outerpillar. As a first step, we pick the outerpath $G \subseteq G^{\prime}$ that is obtained by removing all but the two outermost degree- 2 vertices of $G^{\prime}$; see Fig. 6 Let $P$ be the path that is dual to $G$. The path $P$ splits the boundary of the outer face of $G$ into two paths $\pi_{1}=u_{1}, \ldots, u_{k}$ and $\pi_{2}=v_{1}, \ldots, v_{\ell}$ that are internally disjoint, and that share exactly their endpoints, i.e., $u_{1}=v_{1}$ and $u_{k}=v_{\ell}$, both of which have degree 2 in $G$. Let $H$ be larger than the maximum weight, and let $W$ denote the total weight of all internal edges. Construct a $2 \mathrm{H} \times W$ box, split it horizontally into two boxes of size $H \times W$. We then split the upper box into $k-2$ rectangles $P\left(u_{2}\right), \ldots, P\left(u_{k-1}\right)$ such 

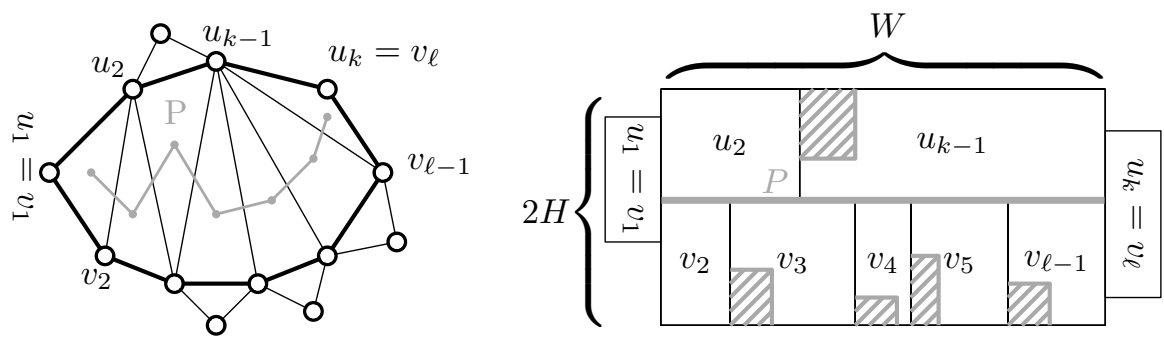

Fig. 6. Construction of an EPRR with 6-gons for outerpillars. Input graph with an outerpath subgraph, drawn with a thick boundary (left). An EPRR of the outerpath with U-shapes for the remaining vertices shown as tiled rectangles (right).

that the width of $P\left(u_{i}\right)$ is the sum of the weights of all internal edges of $G$ incident to $u_{i}$. We split the lower box into boxes for $v_{2}, \ldots, v_{\ell-1}$ analogously; see Fig. 6 Observe that this ensures correct contact lengths for all internal edges of $G$. Next, we place rectangles for $u_{1}$ and $u_{k}$ as boxes to the left and right of the drawing such that they have the correct contact lengths. This ensures correct contact lengths except for internal vertices of $\pi_{1}$ and $\pi_{2}$, respectively, that are adjacent. They touch in a segment of length $H$, which is too long. To remedy this, we remove for any such pair $v_{i} v_{i+1}$ occurring in this order on $\pi_{1}$ or $\pi_{2}$ a corner of the rectangle of $v_{i+1}$. This corner is chosen such that its width is at most half the smallest contact length, and such that afterwards $\left|s\left(v_{i} v_{i+1}\right)\right|=\omega\left(v_{i} v_{i+1}\right)$. This finishes the construction for outerpaths. For the more general case of outerpillars observe that there is a small U-shaped gap between any adjacent pair of vertices on the outer face, and we can hence use the same approach as in the proof of Proposition 2 to attach further leaves to the central outerpath determined by the spine $P$. Note that the polygons can be stretched such that one contact has the correct length, so that only six bends are necessary.

This completely characterizes the complexity of length-universal layouts for internally triangulated graphs. As we have seen outerplanar graphs whose dual is a caterpillar require complexity 6 . The dual of the example graph showing that the 8 bends are necessary is a lobster. Hence our results are best possible.

One disadvantage is that our drawings have an outer face of high complexity. However, we can show that one cannot do better. If one limits the complexity of the outer face to some fixed number $K$, then there exist outerplanar graphs that require complexity $k_{0}$ for any positive integer $k_{0}>K$.

Acknowledgment. This work was partly supported by GRADR - EUROGIGA project no. 10-EuroGIGA-OP-003 and by the ,Concept for the Future' of KIT under project YIG 10-209 within the framework of the German Excellence Initiative.

\section{References}

1. Alam, M.J., Biedl, T., Felsner, S., Gerasch, A., Kaufmann, M., Kobourov, S.G.: LinearTime Algorithms for Hole-Free Rectilinear Proportional Contact Graph Representations. In: Asano, T., Nakano, S.-i., Okamoto, Y., Watanabe, O. (eds.) ISAAC 2011. LNCS, vol. 7074, pp. 281-291. Springer, Heidelberg (2011) 
2. Alam, M.J., Biedl, T., Felsner, S., Kaufmann, M., Kobourov, S.G., Ueckerdt, T.: Computing cartograms with optimal complexity. In: Proc. 28th ACM Symp. on Computational Geometry, SoCG 2012, pp. 21-30. ACM (2012)

3. Battista, G.D., Eades, P., Tamassia, R., Tollis, I.G.: Graph Drawing: Algorithms for the Visualization of Graphs. Prentice Hall (1999)

4. de Berg, M., Mumford, E., Speckmann, B.: On rectilinear duals for vertex-weighted plane graphs. Discrete Mathematics 309(7), 1794-1812 (2009)

5. Biedl, T., Genc, B.: Complexity of octagonal and rectangular cartograms. In: Proc. 17th Canadian Conference on Computational Geometry, CCCG 2005, pp. 117-120 (2005)

6. Biedl, T., Ruiz Velázquez, L.E.: Orthogonal Cartograms with Few Corners Per Face. In: Dehne, F., Iacono, J., Sack, J.-R. (eds.) WADS 2011. LNCS, vol. 6844, pp. 98-109. Springer, Heidelberg (2011)

7. Cabello, S., Demaine, E.D., Rote, G.: Planar embeddings of graphs with specified edge lengths. J. Graph Algorithms Appl. 11(1), 259-276 (2007)

8. Chalopin, J., Gonçalves, D.: Every planar graph is the intersection graph of segments in the plane. In: Proc. 41st Ann. ACM Symp. Theory of Computing, STOC 2009, pp. 631-638. ACM (2009)

9. Eades, P., Wormald, N.C.: Fixed edge-length graph drawing is NP-hard. Discrete Applied Mathematics 28(2), 111-134 (1990)

10. Eppstein, D., Mumford, E., Speckmann, B., Verbeek, K.: Area-universal and constrained rectangular layouts. SIAM J. Comput. 41(3), 537-564 (2012)

11. He, X.: On finding the rectangular duals of planar triangular graphs. SIAM J. Comput. 22(6), 1218-1226 (1993)

12. Hliněný, P., Kratochvíl, J.: Representing graphs by disks and balls (a survey of recognitioncomplexity results). Discrete Mathematics 229(1-3), 101-124 (2001)

13. Kawaguchi, A., Nagamochi, H.: Orthogonal Drawings for Plane Graphs with Specified Face Areas. In: Cai, J.-Y., Cooper, S.B., Zhu, H. (eds.) TAMC 2007. LNCS, vol. 4484, pp. 584 594. Springer, Heidelberg (2007)

14. Knuth, D.E., Raghunathan, A.: The problem of compatible representatives. SIAM J. Discrete Math. 5(3), 422-427 (1992)

15. Koebe, P.: Kontaktprobleme der konformen abbildung. Ber. Sächs. Akad. Wiss. Leipzig, Math.-Phys. Klasse 88, 141-164 (1936)

16. Kozminski, K.A., Kinnen, E.: Rectangular dualization and rectangular dissections. IEEE Trans. Circuits and Systems 35(11), 1401-1416 (1988)

17. van Kreveld, M., Speckmann, B.: On rectangular cartograms. Comput. Geom. Theory Appl. 37(3), 175-187 (2007)

18. Leinwand, S.M., Lai, Y.T.: An algorithm for building rectangular floor-plans. In: Proc. 21st Design Automation Conference, pp. 663-664 (1984)

19. Liao, C.C., Lu, H.I., Yen, H.C.: Compact floor-planning via orderly spanning trees. J. Algorithms 48(2), 441-451 (2003)

20. Lichtenstein, D.: Planar formulae and their uses. SIAM J. Comput. 11(2), 329-343 (1982)

21. McKee, T.A., McMorris, F.R.: Topics in Intersection Graph Theory. SIAM (1999)

22. Nishizeki, T., Rahman, M.S.: Rectangular drawing algorithms. In: Tamassia, R. (ed.) Handbook of Graph Drawing and Visualization, ch. 13. CRC Press (2013) (to appear)

23. Tamassia, R.: Drawing algorithms for planar st-graphs. Australasian J. Combinatorics 2, 217 235 (1990)

24. Yeap, K.H., Sarrafzadeh, M.: Floor-planning by graph dualization: 2-concave rectilinear modules. SIAM J. Comput. 22(3), 500-526 (1993) 